\title{
Role of the ventral medial prefrontal cortex in mediating behavioral control-induced reduction of later conditioned fear
}

\author{
Michael V. Baratta, ${ }^{1}$ Thomas R. Lucero, Jose Amat, Linda R. Watkins, \\ and Steven F. Maier \\ Department of Psychology and Center for Neuroscience, University of Colorado, Boulder, Colorado 80309-0345, USA
}

\begin{abstract}
A prior experience of behavioral control over a stressor interferes with subsequent Pavlovian fear conditioning, and this effect is dependent on the activation of the ventral medial prefrontal cortex (mPFCv) at the time of the initial experience with control. It is unknown whether mPFCv activity is necessary during fear learning and/or testing for this interference to occur. One week following controllable stress, the infralimbic cortex (IL) was temporarily inactivated either before fear learning or later testing. Inactivation of the IL before the test for conditioned fear, but not before conditioning, blocked the fear reducing effects of prior controllable stress. This suggests that the experience with control interferes with the expression of fear behavior and not the learning of the association, and that the $\mathrm{mPFCv}$ is needed to regulate conditioned fear behavior.
\end{abstract}

A previous experience with stressors over which the subject has behavioral control (the ability to influence the onset, termination, intensity, or pattern of the stressor) can blunt the typical behavioral responses to subsequent stressors that are uncontrollable, a process labeled "behavioral immunization" (Seligman and Maier 1967). For example, rats exposed to controllable escapable tail shocks (ES) later fail to show the typical behavioral outcomes of exposure to uncontrollable yoked inescapable tail shocks (IS), such as a deficit in shuttle-box escape behavior or exaggerated shock-elicited freezing (Seligman and Maier 1967; Williams and Maier 1977; Amat et al. 2006). Additionally, recent experiments have found that an initial experience with ES blunts some of the typical neural responses to subsequent IS (Amat et al. 2006). For example, prior ES blocked the intense activation of serotonergic cells (5-HT) in the dorsal raphe nucleus (DRN) at the time of IS $1 \mathrm{wk}$ later, thereby providing a neural mechanism for behavioral immunization. Intense 5-HT activation within the DRN is normally produced by IS and is critical to the production of the behavioral effects of IS (Maier et al. 1995; Grahn et al. 1999). Furthermore, the immunizing effects of ES are dependent on the activation of the ventral medial prefrontal cortex (mPFCv) both at the time of the initial experience with control and at the time of subsequent uncontrollable stress (Amat et al. 2006). The pattern of data suggests that the presence of control activates the mPFCv, which in turn exerts inhibitory control over the DRN (Amat et al. 2005). Furthermore, the activation of the mPFCv by an initial experience with control seems to "tie" mPFCv activation to some aspect of the stressor so that later uncontrollable stressors now activate mPFCv regulation of the DRN.

The mPFCv also projects to the amygdala. The projections and their functions are complex (Vidal-Gonzalez et al. 2006), but it is clear that the infralimbic region (IL) sends glutamatergic projections that synapse onto GABAergic interneurons in the intercalated cell region (ITC) that inhibit the output of the central nucleus of the amygdala (CeA) (Quirk et al. 2003; Berretta et al. 2005). Since the CeA mediates the expression of the conditioned

1 Corresponding author.

E-mail michael.baratta@colorado.edu; fax (303) 492-2967.

Article is online at http://www.learnmem.org/cgi/doi/10.1101//m.800308. fear response through its projections to brainstem and hypothalamic sites (LeDoux et al. 1988), stimulation of the IL may be expected to inhibit fear responses, and this appears to be the case (Milad and Quirk 2002).

The two sets of findings reviewed above led Baratta et al. (2007) to examine the impact of ES and yoked IS on contextual and auditory fear conditioning occurring $7 \mathrm{~d}$ later in a different environment. Not surprisingly, prior IS facilitated the fear response to conditioned stimuli (CS) measured $24 \mathrm{~h}$ after the conditioning. More interestingly, prior ES potently reduced later fear conditioning. The reduction in fear conditioning by ES is a striking finding as ES is quite "stressful" (Maier et al. 1986) and serves as an effective unconditioned stimulus (US) to condition fear to cues that are present (Mineka and Hendersen 1985). Certainly, ES is not "negatively fearful." Analogously to the data reported by Amat et al. (2006), inactivation of the MPFCv during ES blocked the ability of ES to reduce subsequent contextual and auditorycued conditioned fear (Baratta et al. 2007).

The amount of fear measured during behavioral testing is commonly attributed to the strength of the association between the CS and US. However, the amount of fear behavior could also be determined by the strength of fear expression to a given level of conditioning. This distinction can be clarified by considering the functions of the amygdala in fear conditioning. The association between the CS and US is thought to be formed in the lateral amygdala (Fendt and Fanselow 1999). The lateral amygdala then projects, either directly or via the basal nucleus to the CeA, with the CeA innervating the proximate mediators of fear responses, likely the periaqueductal gray in the case of freezing (Kim et al. 1993). Clearly, a process that alters the level of output of the CeA directly, rather than by modulating the lateral or basolateral amygdala (BLA), would alter the expression of fear responses, but not the CS-US association.

Indeed, data reported by Baratta et al. (2007) suggest that the prior experience of control may reduce the later expression of fear rather than the conditioning itself. Rats were exposed to ES or yoked IS $24 \mathrm{~h}$ after conditioning. When later tested, the rats that had been given ES showed reduced fear by the second minute of testing. It is unlikely that ES somehow acted on the conditioning that had occurred $24 \mathrm{~h}$ earlier. 


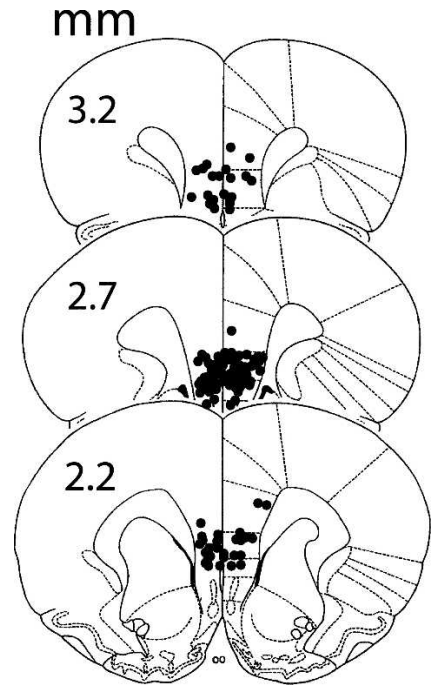

Figure 1. Microinjection cannula placements in IL. The black circles represent the injector-tip placements for rats in all experiments. Numerals indicate rostral distance from bregma $(\mathrm{mm})$.

If experiencing ES reduces the expression of conditioned fear, and if activation of IL-induced inhibition of CeA output is the mediating mechanism, then inhibiting the IL during fear testing should remove the fear reduction produced by the prior ES. That is, if ES does not reduce the association formed between CS and US but only the expression of fear, then IL inhibition should reveal the fear that had been conditioned, but not expressed. Conversely, IL inhibition during the conditioning should not influence the fear reduction produced by prior ES since the associative process is not altered by the ES. The present experiments test these possibilities.

The first experiment was designed to determine if activity in the IL during fear acquisition is necessary for ES-induced reduction in fear to occur. It is possible that in ES subjects IL output to the amygdala during acquisition disrupts the associative strength between the US and the CS. We tested this hypothesis by inactivating the IL with muscimol, a potent $\mathrm{GABA}_{\mathrm{A}}$ agonist, before acquisition. Bilateral microinjection cannulae $(2.6 \mathrm{~mm}$ rostral, $\pm 0.5 \mathrm{~mm}$ lateral, $3.6 \mathrm{~mm}$ ventral to bregma) were implanted. Two weeks later, male Sprague-Dawley rats (Harlan) were exposed to either ES or yoked IS, which consisted of 100 trials of tail shock $(30 \times 1.0 \mathrm{~mA}, 30 \times 1.3 \mathrm{~mA}, 40 \times 1.6 \mathrm{~mA})$ with an average 60 -sec intertrial interval. Tail shock was terminated for both rats when the ES rat met the escape requirement (turning a small wheel mounted on the front of the chamber). Thus, the intensity and the duration of the tail shocks were identical for each rat in the pair. Nonshocked home cage (HC) rats remained undisturbed in the colony. One week later, rats received bilateral microinjections of muscimol $(0.25 \mu \mathrm{L} / 250 \mathrm{ng} /$ side $)$ or saline vehicle into the IL $1 \mathrm{~h}$ prior to contextual and cued fear conditioning (Fig. 1). Animals were allowed to explore the conditioning chamber, a different environment from that in which tail shock occurred, for $2 \mathrm{~min}$ and then presented with a $15-\mathrm{sec}$ tone $(76 \mathrm{~dB} ; 2 \mathrm{kHz})$ immediately followed by a single 2 -sec foot shock (1.0 mA). The following day, rats were re-exposed to the conditioned context and tone, and freezing was assessed as a measure of conditioned fear. In all experiments, the rats were counterbalanced so that half received the context test first and the other half received the tone test first. Also, in order to eliminate the contribution of contextual fear conditioning to freezing to the tone, the tone test was conducted in a novel environment. The context fear test consisted of a 5-min session. In the tone test, freezing was first measured for $3 \mathrm{~min}$ in the new context in the absence of the tone and then for 3 min during the tone.

As in Baratta et al. (2007) there was no freezing on the day of conditioning, indicating that fear does not generalize from the ES/IS to fear-conditioning environments. Treatment with muscimol prior to acquisition had no effect on group freezing levels to the context or tone on the test day (Fig. 2). As is typical, prior IS potentiated fear conditioning, whereas prior ES led to a reduction. Percentage of freezing scores ( $n=6-8$ /group) to the context is shown in Fig. 2A. ANOVA revealed a significant main effect for stress condition $\left(F_{(2,37)}=63.207, P<0.05\right)$ but not for drug $\left(F_{(1,37)}=0.346, P=0.559\right)$.

The pattern of freezing responses to the conditioned auditory-cue was identical to that of the conditioned context. Percentage of freezing scores ( $n=6-8 /$ group) to the novel environment (pretone) and to the conditioned auditory cue (tone) is shown in Fig. 2B. A repeated-measures ANOVA revealed a significant main effect for stress condition $\left(F_{(2,37)}=37.712, P<0.05\right)$, tone condition $\left(F_{(2,37)}=239.95, P<0.05\right)$, and the interaction between stress and tone conditions $\left(F_{(2,37)}=25.36, P<0.05\right)$. However, muscimol treatment had no effect in any of the groups on the development of freezing responses to the conditioned tone.

Experiment 2 sought to determine if activity in IL during fear expression is necessary for ES-induced interference with later fear conditioning. The procedures followed were identical to those of Experiment 1 except muscimol or vehicle was now microinjected into IL $1 \mathrm{~h}$ prior to the test for conditioned fear. Additionally, in order to prevent multiple infusions of muscimol on the same day, assessment for conditioned fear to the tone and to the context was separated by $24 \mathrm{~h}$.

Inactivation of IL prior to the test for conditioned fear increased the level of freezing in ES subjects to both context and tone (Fig. 3). In vehicle-treated subjects, prior ES and IS lead to a bidirectional modulation of conditioned fear, replicating previous results (Baratta et al. 2007). However, muscimol treatment selectively blocked the ES-induced reduction of the conditioned fear response such that now ES rats expressed a level of fear to the context and to the tone similar to that of $\mathrm{HC}$ and IS. Percentage
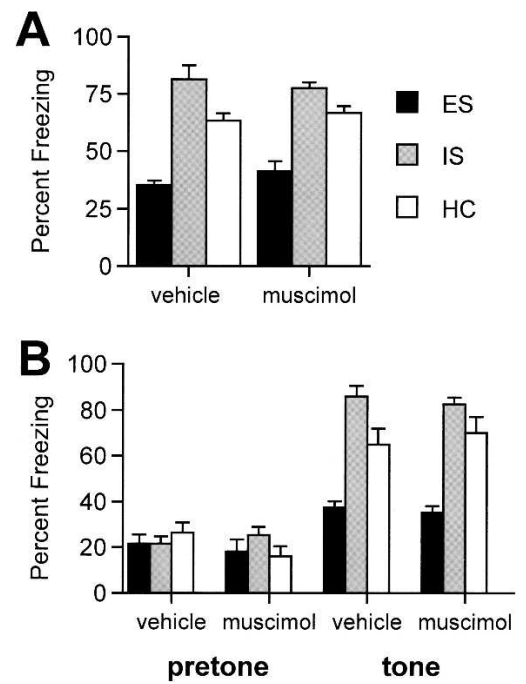

Figure 2. Inactivation of IL before conditioning does not prevent ESinduced resistance to later conditioned fear. $(A)$ Mean percent freezing in the conditioning context for groups given ES, IS, or HC 1 wk before fear conditioning. $(B)$ Mean percent freezing to the novel test environment (pretone) and to the tone CS for groups given ES, IS, or HC 1 wk before fear conditioning. Muscimol or vehicle was microinjected into the IL $1 \mathrm{~h}$ before fear acquisition. 

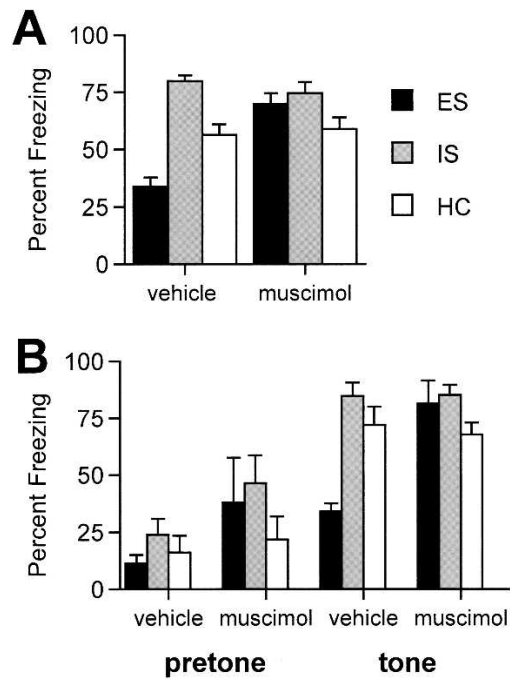

Figure 3. Inactivation of IL before test for conditioning does prevent ES-induced resistance to later conditioned fear. $(A)$ Mean percent freezing in the conditioning context for groups given ES, IS, or HC 1 wk before fear conditioning. (B) Mean percent freezing to the novel test environment (pretone) and to the tone CS for groups given ES, IS, or $\mathrm{HC} 1 \mathrm{wk}$ before fear conditioning. Muscimol or vehicle was microinjected into the IL $1 \mathrm{~h}$ before test for conditioned fear.

of freezing scores ( $n=6-9 /$ group) to the context is shown in Fig. $3 \mathrm{~A}$. ANOVA revealed a significant main effect for stress condition $\left(F_{(2,39)}=16.108, P<0.05\right)$, drug $\left(F_{(1,39)}=8.568, P<0.05\right)$, and an interaction between stress condition and drug $\left(F_{(2,39)}=10.577\right.$, $P<0.05)$. The main effect for group reflects the same pattern as Experiment 1 in which vehicle-injected IS and ES rats respectively increased and decreased freezing compared to HC (Fisher's PLSD, $P \mathrm{~s}<0.05)$. The main effect of drug and the stress condition by drug interaction reflects a selective increased freezing to the conditioned context in muscimol-injected ES subjects.

The effect of intra-IL muscimol on freezing in ES subjects was similar for the conditioned tone (Fig. 3B). A repeated measures ANOVA revealed a significant main effect for stress condition $\left(F_{(2,39)}=4.332, P<0.05\right)$, drug $\left(F_{(1,39)}=8.666, P<0.05\right)$, tone condition $\left(F_{(1,39)}=96.809, P<0.05\right)$, and the interaction between stress condition and drug $\left(F_{(2,39)}=3.597, P<0.05\right)$. It should be noted that muscimol treatment tended to increase freezing in the pretone condition for groups that received prior tail shock, but this enhancement was not statistically significant. For tone-conditioned freezing, post-hoc Fisher's PLSD tests indicated that ES-muscimol did not differ from HC-muscimol, HCvehicle, IS-muscimol, or IS-vehicle but did differ from ESvehicle. Thus, activity in IL during fear expression is necessary for the interfering effects of prior ES.

These results suggest that prior ES interferes with later conditioned fear responses through a reduction in fear expression and not through a disruption in the formation of fear memories. Notably, inactivation of the IL prior to test for conditioning eliminated the difference between ES and the other groups by selectively increasing the freezing response of the ES group. The argument here is that inactivation of the IL in ES subjects during fear expression "unmasks" the fear learning that had occurred in this group. It is unlikely that muscimol before the test for conditioning would selectively increase the fear response in ES subjects if they had not acquired the association between the US and CS the previous day. In support of this, intra-IL muscimol infusion prior to acquisition had no effect on the ability of ES to reduce fear expression the next day.

The present results fit well with previous studies exploring the role of the mPFCv in fear-related processes. Pharmacological inactivation or pretraining lesions of the mPFCv does not impair acquisition of conditioned fear (Morgan et al. 1993; Quirk et al. 2000; Sierra-Mercado et al. 2006), and IL muscimol microinjection before conditioning here did not alter conditioning. Rather, recent work from Quirk and colleagues (e.g., Vidal-Gonzalez et al. 2006) has implicated the mPFCv in the expression of conditioned fear with the IL being the critical region within the mPFCV that inhibits amygdala function (Milad and Quirk 2002). Thus, the current data suggest that experiencing behavioral control over a stressor alters the mPFCv in such a way that later exposure to conditioned fear stimuli during testing now activates the IL, resulting in a reduction in amygdala-dependent fear expression. Interestingly, ES-induced resistance to fear-related processes is restricted to learned fear and not innate fear (Baratta et al. 2007) whose expression may not be dependent on the CeA (Fendt et al. 2003).

It is currently unclear how the IL mediates ES interference with fear expression. Given that mPFCv projection neurons have sparse projections to the output region of the CeA (McDonald 1998), it is likely that IL inhibition of the conditioned fear response is through an indirect route. One possibility is that the IL selectively targets local inhibitory cells that dampen the BLA input to the CeA. The GABAergic ITC cells of the amygdala receive robust projections from the IL (McDonald et al. 1996; Freedman et al. 2000). This interconnected network of cells, positioned between the BLA and CeA, sends projections that synapse onto the dendrites of CeA neurons and inhibits them (Royer et al. 1999). In addition, stimulating the IL has been shown to increase the activity of ITC cells as detected by Fos protein (Berretta et al. 2005). This suggests that the IL can modulate amygdaladependent conditioned fear responses through its projections to ITC cells. Thus, the present data suggest that an initial experience with control may alter the IL in such a way that subsequent exposure to conditioned fear stimuli now activates IL output to the ITC region and inhibits fear expression.

In a more general sense, the present data caution against interpreting instances of the modulation of fear conditioning as necessarily representing alterations in the associative/memory formation process. It is always possible, as has proven to be the case with the effects of experiencing control, that a fear-altering manipulation influences response expression rather than learning. With regard to the mPFCv, it may be generally true that it modulates fear expression rather than fear learning.

\section{References}

Amat, J., Baratta, M.V., Paul, E., Bland, S.T., Watkins, L.R., and Maier, S.F. 2005. Medial prefrontal cortex determines how stressor controllability affects behavior and dorsal raphe nucleus. Nat. Neurosci. 8: 365-371.

Amat, J., Paul, E., Zarza, C.M., Watkins, L.R., and Maier, S.F. 2006. Previous experience with behavioral control over stress blocks the behavioral and dorsal raphe nucleus activating effects of later uncontrollable stress: Role of the ventral medial prefrontal cortex. $J$. Neurosci. 26: 13264-13272.

Baratta, M.V., Christianson, J.P., Gomez, D.M., Zarza, C.M., Amat, J., Masini, C.V., Watkins, L.R., and Maier, S.F. 2007. Controllable versus uncontrollable stressors bi-directionally modulate conditioned but not innate fear. Neuroscience 146: 1495-1503.

Berretta, S., Pantazopoulos, H., Caldera, M., Pantazopoulos, P., and Pare, D. 2005. Infralimbic cortex activation increases c-Fos expression in intercalated neurons of the amygdala. Neuroscience 132: 943-953.

Fendt, M. and Fanselow, M.S. 1999. The neuroanatomical and neurochemical basis of conditioned fear. Neurosci. Biobehav. Rev. 23: $743-760$.

Fendt, M., Endres, T., and Apfelbach, R. 2003. Temporary inactivation of the bed nucleus of the stria terminalis but not of the amygdala blocks freezing induced by trimethylthiazoline, a component of fox feces. J. Neurosci. 23: 23-28.

Freedman, L.J., Insel, T.R., and Smith, Y. 2000. Subcortical projections of 
area 25 (subgenual cortex) of the macaque monkey. J. Comp. Neurol. 421: $172-188$.

Grahn, R.E., Will, M.J., Hammack, S.E., Maswood, S., McQueen, M.B. Watkins, L.R., and Maier, S.F. 1999. Activation of serotonin-immunoreactive cells in the dorsal raphe nucleus in rats exposed to an uncontrollable stressor. Brain Res. 826: 35-43.

Kim, J.J., Rison, R.A., and Fanselow, M.S. 1993. Effects of amygdala, hippocampus, and periaqueductal gray lesions on short- and long-term contextual fear. Behav. Neurosci. 107: 1093-1098.

LeDoux, J.E., Iwata, J., Cicchetti, P., and Reis, D.J. 1988. Different projections of the central amygdaloid nucleus mediate autonomic and behavioral correlates of conditioned fear. J. Neurosci. 8: $2517-2529$.

Maier, S.F., Ryan, S.M., Barksdale, C.M., and Kalin, N.H. 1986. Stressor controllability and the pituitary-adrenal system. Behav. Neurosci. 100: 669-674.

Maier, S.F., Grahn, R.E., and Watkins, L.R. 1995. 8-OH-DPAT microinjected in the region of the dorsal raphe nucleus blocks and reverses the enhancement of fear conditioning and interference with escape produced by exposure to inescapable shock. Behav. Neurosci. 109: 404-412.

McDonald, A.J. 1998. Cortical pathways to the mammalian amygdala. Prog. Neurobiol. 55: 257-332.

McDonald, A.J., Mascagni, F., and Guo, L. 1996. Projections of the medial and lateral prefrontal cortices to the amygdala: A Phaseolus vulgaris leucoagglutinin study in the rat. Neuroscience 71: 55-75.

Milad, M.R. and Quirk, G.J. 2002. Neurons in medial prefrontal cortex signal memory for fear extinction. Nature 420: $70-74$.

Mineka, S. and Hendersen, R.W. 1985. Controllability and predictability in acquired motivation. Annu. Rev. Psychol. 36: 495-529.
Morgan, M.A., Romanski, L.M., and LeDoux, J.E. 1993. Extinction of emotional learning: Contribution of medial prefrontal cortex. Neurosci. Lett. 163: 109-113.

Quirk, G.J., Russo, G.K., Barron, J.L., and Lebron, K. 2000. The role of ventromedial prefrontal cortex in the recovery of extinguished fear I. Neurosci. 20: 6225-6231.

Quirk, G.J., Likhtik, E., Pelletier, J.G., and Pare, D. 2003. Stimulation of medial prefrontal cortex decreases the responsiveness of central amygdala output neurons. J. Neurosci. 23: 8800-8807.

Royer, S., Martina, M., and Pare, D. 1999. An inhibitory interface gates impulse traffic between the input and output stations of the amygdala. J. Neurosci. 19: 10575-10583.

Seligman, M.E. and Maier, S.F. 1967. Failure to escape traumatic shock. J. Exp. Psychol. 74: 1-9.

Sierra-Mercado Jr., D., Corcoran, K.A., Lebron-Milad, K., and Quirk, G.J. 2006. Inactivation of the ventromedial prefrontal cortex reduces expression of conditioned fear and impairs subsequent recall of extinction. Eur. J. Neurosci. 24: 1751-1758.

Vidal-Gonzalez, I., Vidal-Gonzalez, B., Rauch, S.L., and Quirk, G.J. 2006. Microstimulation reveals opposing influences of prelimbic and infralimbic cortex on the expression of conditioned fear. Learn. Mem. 13: 728-733.

Williams, J.L. and Maier, S.F. 1977. Transsituational immunization and therapy of learned helplessness in the rat. J. Exp. Psychol. Anim. Behav. Process. 3: 240-255.

Received October 10, 2007; accepted in revised form December 10, 2007. 


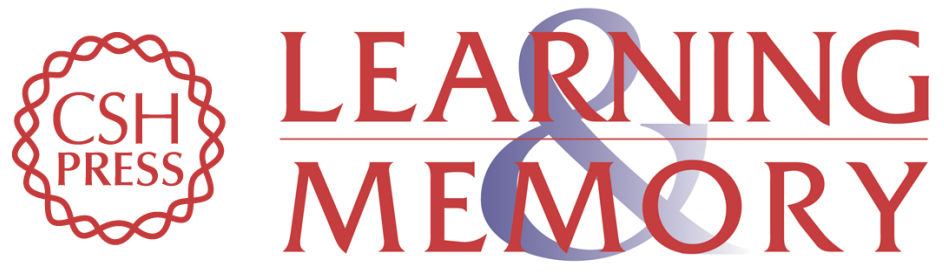

\section{Role of the ventral medial prefrontal cortex in mediating behavioral control-induced reduction of later conditioned fear}

Michael V. Baratta, Thomas R. Lucero, Jose Amat, et al.

Learn. Mem. 2008, 15:

Access the most recent version at doi:10.1101//m.800308

References This article cites 24 articles, 7 of which can be accessed free at:

http://learnmem.cshlp.org/content/15/2/84.full.html\#ref-list-1

License

Email Alerting Receive free email alerts when new articles cite this article - sign up in the box at the Service top right corner of the article or click here. 